\title{
LA PHOTOGRAPHIE COMME SOURCE POUR L'HISTORIEN
}

\section{Laurence van Ypersele ${ }^{1}$}

L'historien a longtemps relégué l'image au rang de source secondaire, loin derrière le document écrit. Pourtant l'image en général peut nous apprendre beaucoup sur les hommes du passé et leur façon d'habiter le monde, sur leur vision de soi et de l'autre, sur leurs désirs et leurs craintes. De tout temps, les hommes ont produit des images. Le monde contemporain, quels que soient les progrès de l'alphabétisation, est un monde d'images. Partout notre regard est sollicité : affiches publicitaires sur les murs de nos villes, cartes postales qui entrent dans les foyers, cinéma, télévision, internet, etc. Au sein de ce monde iconographique, en moins de deux siècles, la photographie s'est taillé une place de choix.

À ce titre, elle est un document incontournable pour l'historien. La prise en compte de la photographie comme source historique a considérablement enrichi l'histoire du colonialisme et l'histoire des guerres contemporaines principalement. Mais il y a bien d'autres domaines à explorer. L'analyse de la photo de presse, par exemple, recèle des possibilités infinies pour étudier les imaginaires collectifs d'une époque : image de la monarchie, image de la femme, image des grèves, etc. L'apport du document photographique pour la recherche historique

1 Professeur à l'Université catholique de Louvain

Recherches en communication, $\mathrm{n}^{\circ} 27$ (2007). 


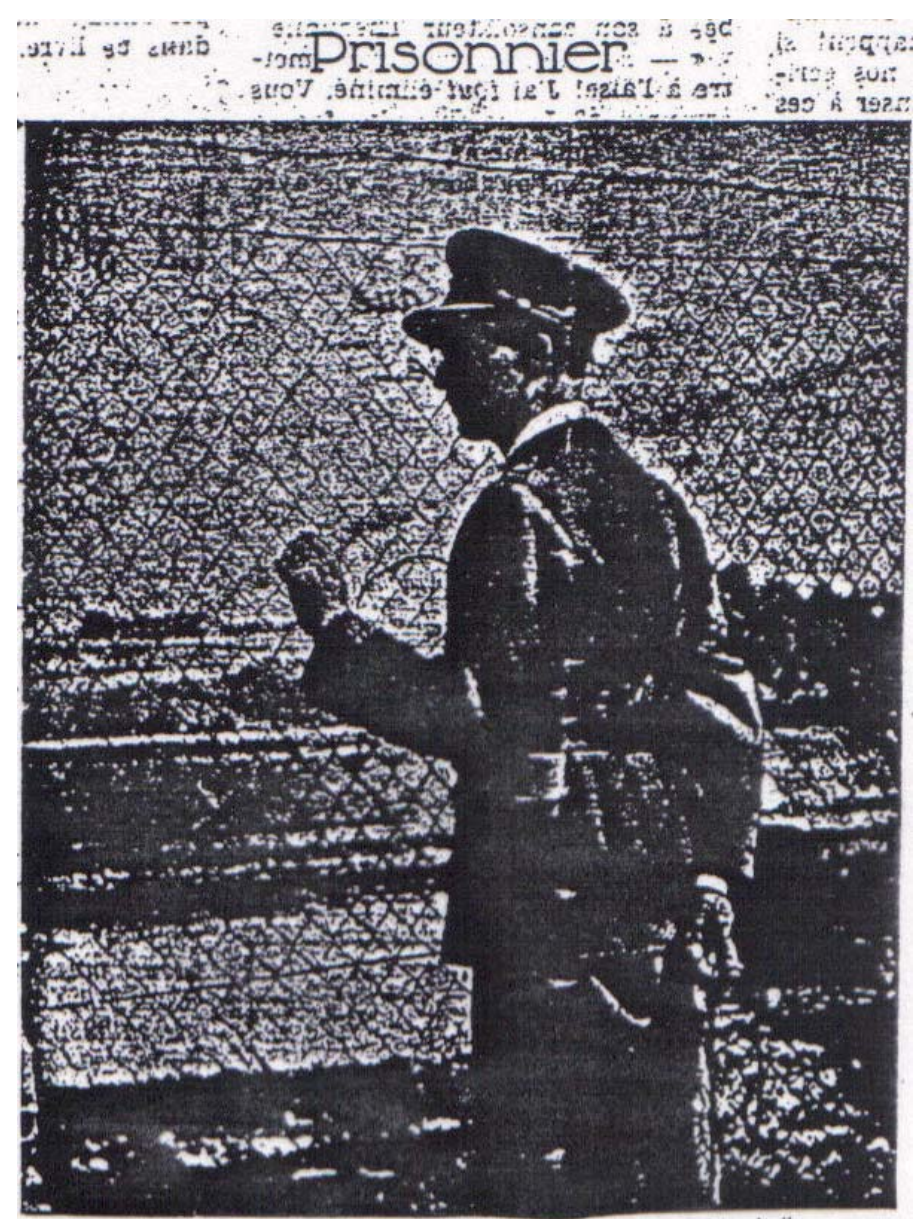

Cetse photo est âuthentique. Le Roi est prisonnier et déporté à Hirschstein. Uné palissade de $3 \mathrm{~m}$. dé haut, faite de treillis et

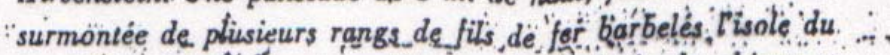
monile extérieur. Il est gardé par $60 \mathrm{SS}$ dotés de chiens et placés sous le commandement dis SS Gruppenfuhrer de Saxe nnn Alvenslehen 
est indéniable. Toutefois son utilisation par l'historien pose toute une série de difficultés. Car, la photographie est ambivalente par nature : plus que tout autre image, elle semble "arrachée" à la réalité, mais en fait elle n'est jamais qu'un point de vue construit sur le monde. La fascination exercée par la photographie dès son invention réside dans cette ambivalence. L'ambition de Niepce était bel et bien de fixer la réalité telle qu'elle est de façon durable. Pour le public, d'ailleurs, la photographie se donne toujours implicitement comme "preuve" du réel, une preuve incontournable et irréfutable ${ }^{1}$. Les journalistes le savent. Ainsi, par exemple, en pleine Question Royale, le journal léopoldiste, Septembre du 22 août 1948, publie une photo du roi prisonnier en Allemagne (fig.1). La légende, qui déborde de loin ce que l'on voit sur l'image, insiste : "Cette photo est authentique. Le Roi est prisonnier et déporté à Hirschstein. Une palissade de $3 \mathrm{~m}$. de haut, faite de treillis et surmontée de plusieurs rangs de fils de fer barbelés, l'isole du monde extérieur. Il est gardé par 60 SS dotés de chiens et placés sous le commandement du SS Gruppenführer de Saxe von Alvensleben"”. Or, comme toute image, la photographie est une construction de la réalité, sans compter qu'elle peut être manipulée, voire falsifiée. Ainsi, la photographie témoigne autant de la perception de la réalité que de la réalité elle-même. L'historien, dans ses analyses, devra toujours tenir compte de cette double dimension.

Pour atteindre à la fois la réalité et l'imaginaire dont témoigne la photographie, l'historien soumet ses documents à une critique rigoureuse. Voyons en quoi elle consiste.

1 C'est ainsi que le lieutenant-colonel français Dupuis, chef du Bureau d'Information à la Presse, définit la photographie, dans une note datée du $1^{\text {er }}$ novembre 1915. Cf. Th. BLONDET-BISCH, "La pratique photographique en France pendant la Grande Guerre", in J.-L. JADOULLE, M. DELWART \& M. MASSON (dir.), L'histoire au prisme de l'image. I. L'historien et l'image fixe, Louvain-la-Neuve, Unité de didactique et de communication en histoire UCL, 2002, p.171.

2 Septembre, 22 août 1948, p. 3 : "Prisonnier". Notons que durant la Question Royale, les léopoldistes utilisent massivement la photographie pour défendre la personne de Léopold III, alors que le camp adverse préfère la caricature (qui, il est vrai, se prête mieux au combat lorsque la polémique est plus idéelle que factuelle). Cf. L. VAN YPERSELE, "La Question Royale ou la guerre des images", in M. DUMOULIN, M. VAN DEN WIJNGAERT \& V. DUJARDIN (dir.), Léopold III, Bruxelles, Complexe, 2001, pp. 303-322. 


\section{Lieu, date et sujet représenté}

Une photo brute sans aucune indication n'est guère utilisable par l'historien. Il faut la dater et identifier le lieu, les personnages, l'événement représenté. Heureusement, la plupart des photos archivées comme celles qui émanent des services photographiques des armées, ainsi que nombre de photos privées dans les albums de famille, sont plus ou moins précisément légendées. Encore faut-il vérifier ces informations, car ces légendes sont parfois erronées volontairement ou non. D'ailleurs, la légende d'une photo fait partie intégrante de l'analyse : la légende peut tantôt se contenter d'identifier les éléments présents dans l'image, tantôt ajouter des informations contextuelles qui ne s'y trouvent pas, tantôt encore émettre une opinion directe ou indirecte qui oriente le regard du lecteur.

\section{Auteur et commanditaires}

Les photographes amateurs ou professionnels peuvent provenir d'horizons bien différents et poursuivre des buts très divers (privé, politique, commercial).

Ainsi, par exemple, les dizaines de milliers de photographies que l'on possède sur le Congo émanent tantôt d'explorateurs ou de membres d'expéditions parfois scientifiques, de militaires au service de l'Etat ou de missionnaires au service de leur congrégation, tantôt de particuliers, d'agents de l'État ou de grandes compagnies, tantôt de photographes professionnels travaillant pour le compte du ministère des Colonies et commanditaire de missions de propagande ${ }^{1}$.

De même, les millions de clichés que l'on possède sur la guerre 1914-1918 émanent tantôt de photographes qui travaillent pour les services photographiques des armées, tantôt de reporters de guerre envoyés par des organes de presse, tantôt d'amateurs civils ou militaires qui ont bravé sans grand problème la censure de guerre.

En effet, si la Grande Guerre marque l'avènement de la propagande de masse par l'image, l'orchestration de cette propagande n'est pas uniquement aux mains de l'État : d'une part, la censure de guerre n'est pas totale et, d'autre part, on compte d'innombrables citoyens, tantôt célèbres tantôt anonymes, qui ont alimenté la propagande patrio-

1 F. MORIMONT \& J.-L. VELLUT, "Clichés d'Afrique au temps de la colonisation belge", in J.-L. JADOULLE, M. DELWART \& M. MASSON (dir.), op. cit., p. 156. 
tique. Les populations n'étaient d'ailleurs pas tout à fait dupes de ces images dont beaucoup ne cachaient ni les grossiers découpages ni les rapprochements impossibles, mais hautement symboliques (comme, par exemple, l'ensemble des chefs d'État alliés réunis sur une même carte postale). En fait, la plupart des gens consentaient au discours iconique sur la guerre, comme ils consentaient à la guerre elle-même. Toutefois, cette production invraisemblable d'images émanait de plusieurs centres, parfois contradictoires, laissant la place à des contre-discours possibles. Alors qu'avec les régimes totalitaires, on entre dans un tout autre univers, puisque la diffusion de l'image est monopolisée par un seul centre et dans un seul but : la tyrannie.

\section{Circonstances dans lesquelles la photo est prise}

Connaître les circonstances dans lesquelles une photo a été prise peut être extrêmement précieux pour l'analyse. Une photo prise sur le vif, dans le feu de l'action, au cœur de l'événement n'a pas le même statut qu'une photo posée dont la mise en scène est réalisée $a$ posteriori à l'aide d'acteurs et de figurants (que ce soit dans une volonté de reconstituer l'événement ou d'apporter une preuve parfaitement fausse). Ainsi, par exemple, après l'incendie du Reichstag, en février 1933, les services nazis diffusèrent une photographie de Marinus Van der Lubbe ${ }^{1}$ tenant en main une boîte d'allumettes pour l'accuser. Or, ces allumettes avaient été placées dans ses mains, par la Gestapo, juste après son arrestation $^{2}$. Sans aller aussi loin, les mises en scène a posteriori peuvent changer ou orienter la signification d'un document, le plus souvent dans un but de propagande. Ainsi, la très célèbre photo (fig.2) des soldats russes hissant le drapeau soviétique sur le Reichstag a été prise, par Yevgeny Khaldei ${ }^{3}$, non pas sur le moment même, mais bien le 2 mai

1 Marinus Van der Lubbe (1909-1934), chômeur, déséquilibré mental et pyromane, d'origine hollandaise. Il fut utilisé et désigné par les nazis comme responsable communiste de l'incendie du Reichstag en février 1933. Arrêté et condamné à mort, il est exécuté en 1934. Cf. S. BERSTEIN \& P. MILZA, Histoire du XX $X^{e}$ siècle, t.1 : 1900-1945, la fin du "monde européen », Paris, Hatier, 1996, p. 317

2 Cité dans Ch. SAMARAN (dir.), L’histoire et ses méthodes, Paris, Gallimard (éditions de La Pléiade), 1961, p. 1390-1391.

3 Yevgeny Khaldei (1917-1998), photographe professionnel ukrainien travaillant pour l'agence TASS de 1935 à 1949, date à laquelle il est licencié en raison de ses origines juives. De 1959 à 1970, il travaille pour la Pravda d'où il est à nouveau licencié pour les mêmes raisons. Cf. P. STEPAN, Icons of Photography. The $20^{\text {th }}$ Century, Munich, 


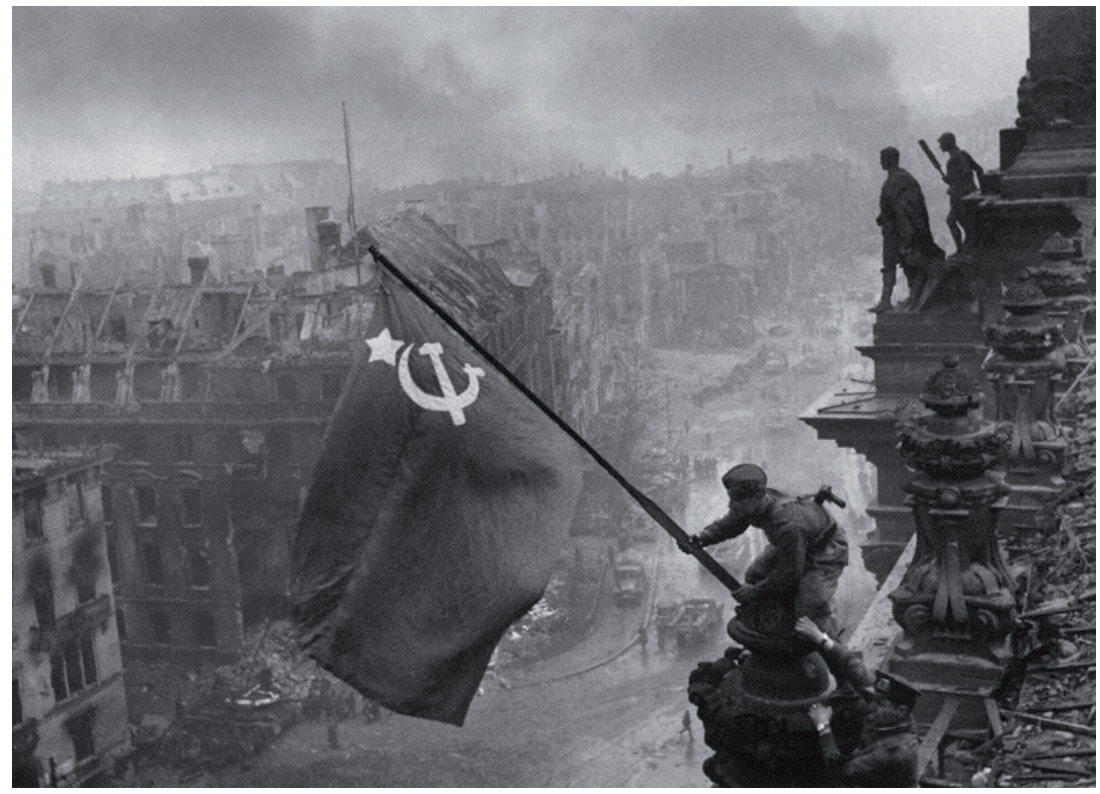

Fig.2. a. Photo du Reichstag, prise par E. Khaldeï, le 2 mai 1945, agence TASS.

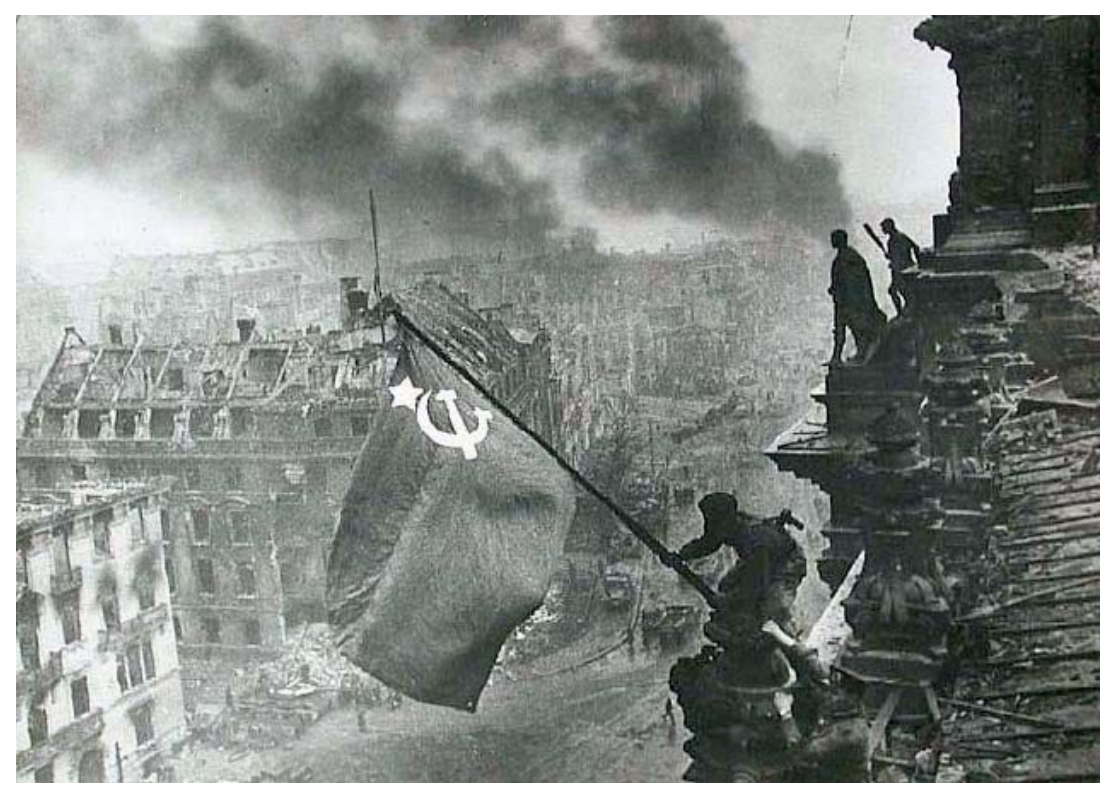

Fig.2. b. Idem, après retouche. 
1945 soit deux jours après la prise de Berlin. Il s'agit d'une mise en scène organisée par le photographe pour l'agence TASS. Cette photo, véritable symbole de la victoire russe, fera le tour du monde, tout en subissant plusieurs modifications. D'une part, certaines versions sont dramatisées : les fumées émanant des ruines de Berlin sont noircies par renforcement des contrastes. D'autre part, un détail, peu visible mais gênant pour les autorités soviétiques, sera effacé : en effet, le soldat qui soutient le drapeau par le dessous possède une montre à chaque bras. Certains pouvant y voir une preuve discrète mais réelle du pillage auquel s'est livrée l'armée rouge, ce détail disparaîtra de l'image un mois après sa première parution, le 3 mai 1945 à Moscou. Et il existe bien d'autres exemples de ce type aussi bien du côté des régimes démocratiques que du côté des régimes totalitaires.

\section{Procédés de construction}

Toute photo est une construction. Mais, elle peut en outre être manipulée et mise au service d'autre chose que ce qu'elle est. Les régimes totalitaires sont passés maîtres dans la falsification des images ${ }^{1} .$. Conscients de l'extraordinaire puissance de l'image sur les masses, ils sont les premiers à pousser aussi loin les techniques de trucage et d'usage de faux. Pour la première fois, la diffusion des images est orchestrée par un seul centre qui rassemble, censure, voire falsifie les documents pour produire ses images au service de $s a$ politique. Désormais, toutes les techniques d'utilisation de l'image sont mises au service d'une idéologie unique et absolue.

À travers cette politique de remaniement systématique des images, les régimes totalitaires ont falsifié l'histoire, manipulé les mémoires et endoctriné les masses.

Pourtant, ces régimes n'ont rien inventé dans le domaine. Dès la fin du XIX ${ }^{\mathrm{e}}$ siècle, on retouche les photos de famille pour supprimer les rides ou les boutons, l'oncle détesté ou le fils qui a mal tourné... Aujourd'hui encore, nos albums de photos ne reprennent que les "bonnes" photos, c'est-à-dire celles qui confortent l'image que nous voulons garder (ou montrer) de nos voyages, de nos fêtes familiales ou de nos amitiés. Mais,

Prestel, 2005, p.96-97.

1 Voir à ce sujet : A. JAUBERT, Le Commissariat aux archives. Les photos qui falsifient l'histoire, Paris, éd. Barrault, 1986. 


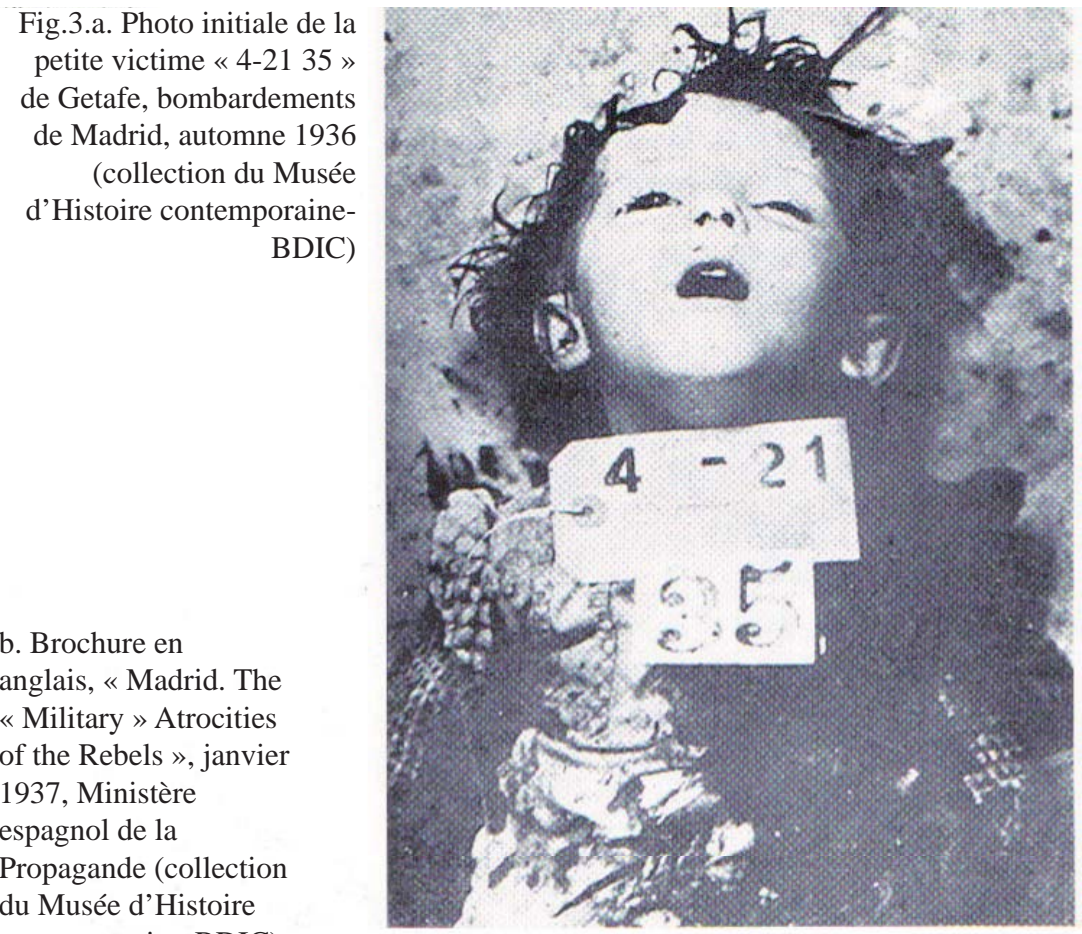
du Musée d'Histoire contemporaine-BDIC)

MADRID

THE "MiLITARY" ATROCITIES OF THE REBElS

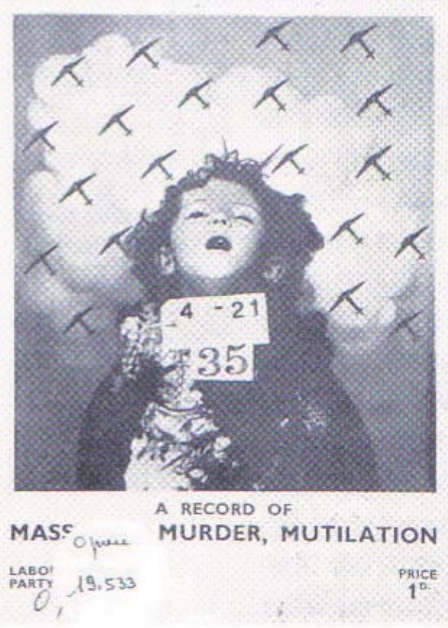

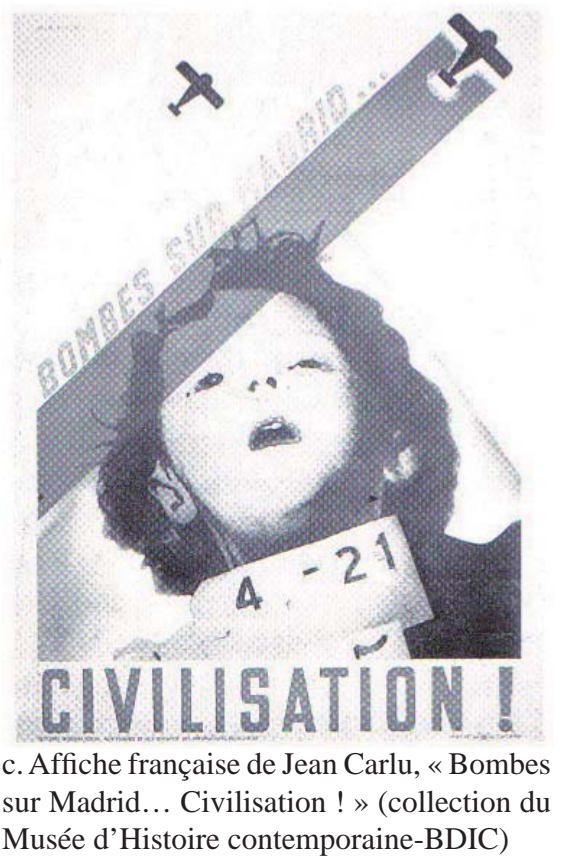


contrairement à d'autres images comme la caricature par exemple, la photo reconstruit la réalité, tout en se donnant pour vraie. Par ailleurs, le rapport à l'authenticité du document a évolué. Au début du XXe siècle, à défaut de reporter sur le terrain, on n'hésite pas à utiliser des images anciennes pour illustrer un nouvel événement, sans intention de manipuler qui que ce soit. Ainsi, par exemple, la couverture de La Semaine Illustrée du 16 août 1914 titre "le roi Albert à la guerre", mais la photo est celle des manœuvres de Beverloo en... juin 1913 ! Enfin, nombre d'affiches de propagande humanitaire visent à sensibiliser les opinions publiques et orienter des comportements sans intention mensongère. Lors de la guerre d'Espagne, par exemple, des affiches républicaines sont des photos montages qui, afin de mobiliser l'opinion internationale, dénoncent les réels massacres de civils par les avions de la légion Condor envoyés par l'Allemagne nazie pour appuyer les nationalistes espagnols. La photo (fig.3) d'une enfant victime des bombardements de Madrid à l'automne 1936, matricule "4-21 35", servira dès janvier 1937 à l'élaboration d'une demi-douzaine d'affiches différentes, légendées aussi bien en anglais qu'en français. L'ajout d'avions derrière la petite victime ou lui barrant le sommet du visage désigne le coupable de cette "atrocité" et de cette injure à la "Civilisation", pour reprendre les termes utilisés dans les légendes ${ }^{1}$.

Cela dit, les exemples d'utilisations fallacieuses de l'image par les démocraties occidentales ne manquent pas. Le cas des images de la guerre du Vietnam est bien connu : suivant l'évolution de l'opinion publique, les premières images ont été nettoyées et affadies, tandis que les dernières seront surchargées et dramatisées ${ }^{2}$. Et que dire de la "guerre chirurgicale" du Golfe, remake en direct d'un Star War de mauvais goût qui a, pourtant, réussi à tenir l'Occident en haleine pendant des semaines ? Plus quotidiennement, nos journaux télévisés sont remplis d'inversions d'images, de recadrages suggestifs, de sélections pour le moins orientées et d'utilisations de séquences de films historiques en

1 Cf. L. GervereaU, Autopsie d'un chef-d'œuvre. Guernica, Paris, Méditerranée, 1996, pp.143-147. Une première affiche émane du Ministère espagnol de la Propagande (version française et anglaise), une autre version française est l'œuvre de Jean Carlu, d'autres encore sont réalisées par Arthur Koestler, par le Comité international aux femmes et aux enfants espagnols et par le Comité mondial contre la guerre et contre le fascisme.

2 Cf. C. LEROY, Under Fire : Great Photographers and Writers in Vietnam, New York, Random House, 2005. 


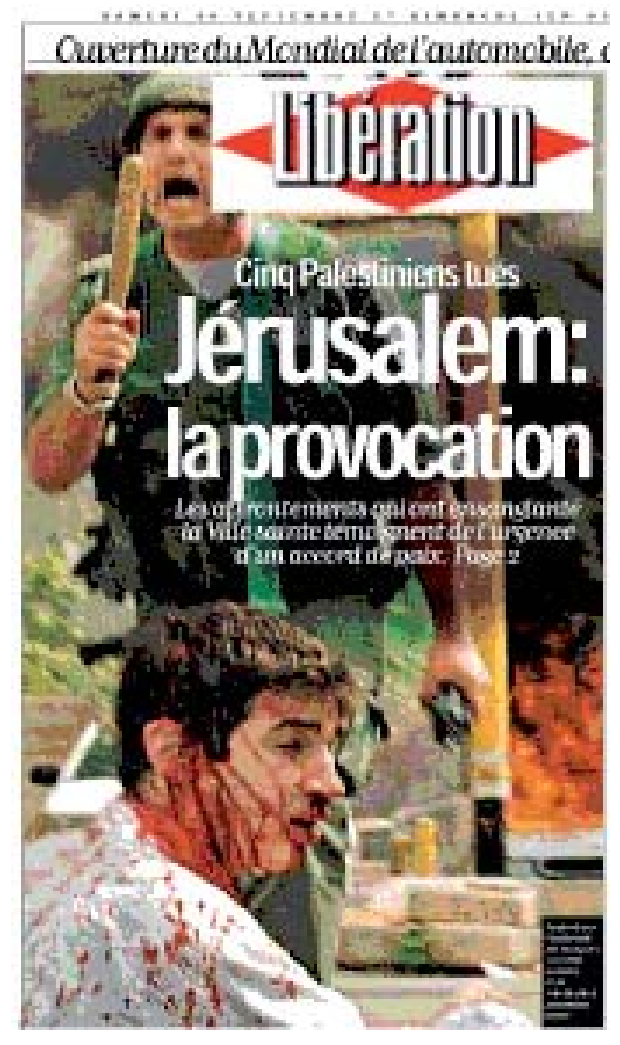

Fig.4.a.Libération,30 septembre2001, p. 1 : « Jérusalem : la provocation».

b. Photo de l'agence Associated Press

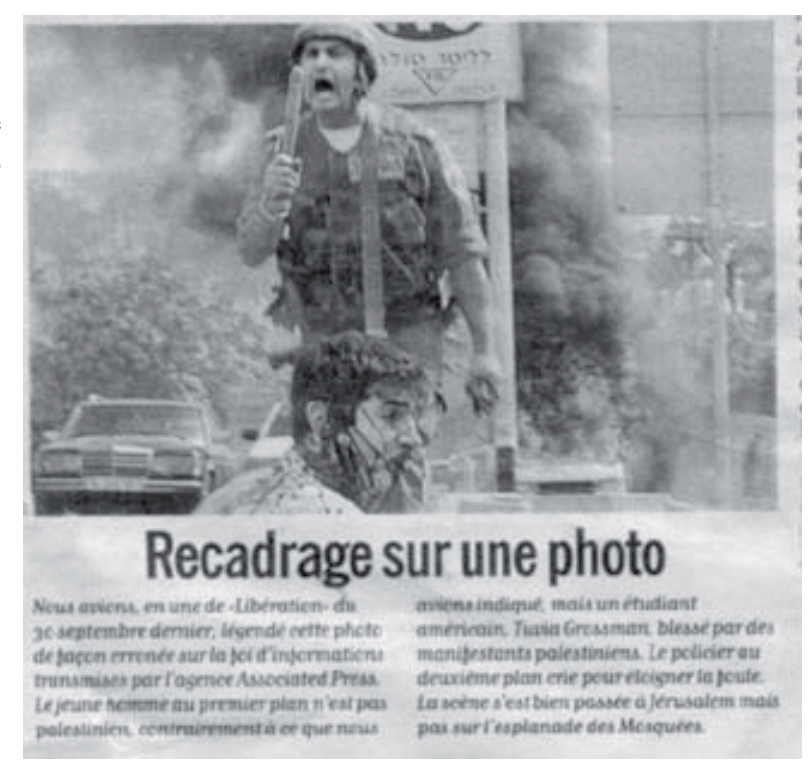


lieu et place de documents d'archives. Sans doute, il s'agit rarement d'images purement et simplement falsifiées ou inventées. Et, si c'est le cas, l'usage fallacieux de l'image est rapidement dénoncé. Ainsi, par exemple, la photo de l'agence Associated Press présentée à la Une de Libération, le 30 septembre 2000, avec pour titre "JÉRUSALEM : la provocation. Vendredi sur l'esplanade des Mosquées, un soldat israélien et un manifestant palestinien blessé" (fig.4), fait immédiatement scandale. Le journal, comme l'agence AP, est d'ailleurs condamné par une Cour parisienne en 2002 à payer 4500 euros de dédommagement à la famille Grossman ${ }^{1}$. En effet, contrairement à ce qu'affirme la légende, cette photo n'a pas été prise sur l'esplanade du Temple où débuta la seconde Intifada, mais près d'une pompe à essence située à la sortie de Jérusalem. Il ne s'agit pas non plus d'une victime palestinienne frappée par un soldat israélien, mais bien d'un étudiant juif américain, Tuvia Grossman, victime d'un attentat palestinien et protégé par un policier israélien d'origine druze ! Quoi qu'il en soit les images choisies et diffusées par les médias façonnent largement l'opinion publique et souvent les manipulent. Il est vrai que le $\mathrm{XX}^{\mathrm{e}}$ siècle apparaît à la fois comme le siècle de l'image et celui de l'avènement des foules.

\section{Public visé}

La question de la réception des images est la plus difficile à résoudre. Mais on peut tout de même s'interroger quant au public visé et au but poursuivi. À cet égard, la nature du document peut être un premier indice : album de famille, publicité commerciale, affiche politique, idéologie du journal. Les rééditions et la publication d'une image sur de multiples supports sont des indices supplémentaires. Ainsi, par exemple, la photo du roi Albert traversant, à cheval, Bruxelles pour se rendre au Palais de la Nation, le 4 août 1914 (fig.5) se retrouve non seulement dans la presse illustrée de l'époque, mais aussi sur carte postale, dans les manuels scolaires et dans de nombreux ouvrages de l'entre-deux-guerres.

Par ailleurs, on peut s'interroger sur le contrat de lecture instauré par l'image avec le public. Ainsi, le rapport que le public entretient avec l'image photographique peut varier du tout au tout : un photomontage apparent joue avec la technique du collage pour étonner ou

1 http://archives.desinfos.com/libe_desinfos.html (consulté, le 28 mai 2007). 


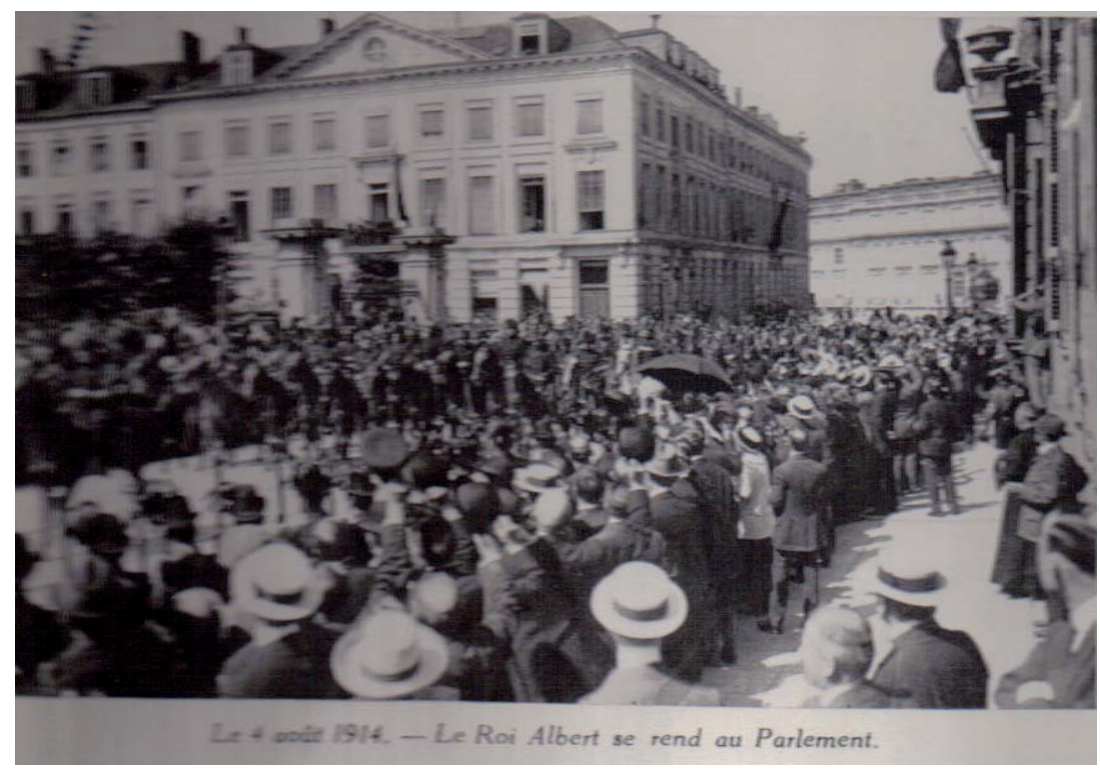

Fig.5. Photo du Roi Albert se rendant à cheval au Palais de la Nation, le 4 août 1914 (dans G. RENCY, Albert, roi des Belges, Bruxelles, Henri Bertels éd., 1934, p.79).

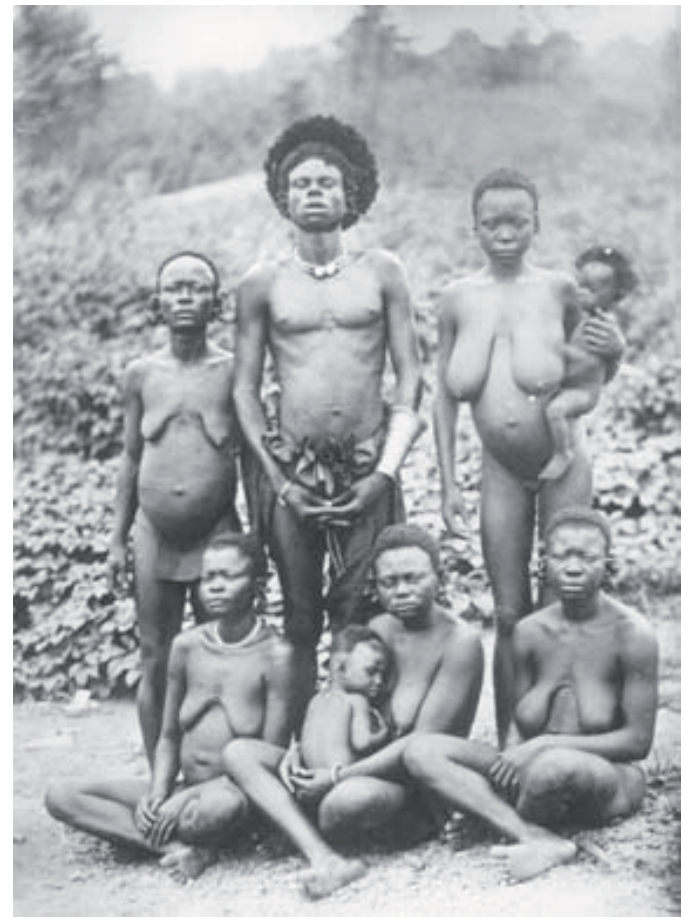

Fig.6. Photo, du Chef Basoko et sa famille, réalisé par le photographe ouest-africain Shanu, ca 1898 (Tervuren, Musée royal de l'Afrique centrale). 
faire rire, alors que la photo de presse s'est toujours donnée pour vraie. En outre, la suspicion à l'égard de la photographie varie dans le temps comme dans l'espace sociologique. Ainsi, la dénonciation du «bourrage de crâne » de la propagande de 1914-1918 durant l'entre-deuxguerres jettera un discrédit durable sur toute dénonciation d'atrocités commises par l'ennemi ${ }^{1}$. L'impact sur la réception de la propagande durant la seconde guerre mondiale et les tentatives de dénonciation de la Solution Finale en découle directement.

\section{Contenu des photographies}

Au plan de la critique interne, l'historien sera aussi bien attentif à l'aspect dénotatif qu'aux connotations qui y sont liées. Il dégagera les différentes thématiques présentes dans ses corpus et pointera les thèmes absents.

Ainsi, pour l'histoire coloniale belge ${ }^{2}$, les dizaines de milliers de clichés que l'on possède permettent, à un premier niveau, d'appréhender une part de la réalité : l'apparence de différentes tribus, les vêtements, l'habitat, la faune et la flore, mais aussi le cérémonial mis en place par les colons, l'acculturation ou l'arrivée des progrès occidentaux en matière scolaire, médicale et technique. À un second niveau, ces mêmes clichés - dont la plupart sont pris par les Européens - témoignent du regard occidental sur l' « autre » et sur « soi »: les photos des tribus (fig.6), généralement des photos de groupe, disent l'étonnement des colons, leur fascination pour l'exotisme et leur désir de classification. Notons que les occidentaux classent les tribus en catégories fermées, alors que la réalité ethnique africaine était beaucoup plus souple ; ce qui aura des répercutions sur l'organisation coloniale : l'imaginaire occidentale transformant la réalité. Tandis que les photos où l'on voit à la

1 Ces dénonciations, comme celles de H. Lasswell et A. Ponsonby, renvoient dos à dos les mensonges ou exagérations des propagandes alliée et allemande, sans s'interroger sur la référence au réel. Or, en ce qui concerne les « atrocités allemandes » commises en août 1914 : la propagande allemande brandit la présence de Francs-Tireurs qui n'existaient pas en 1914 (mais bien en 1870-1871), tandis que la propagande alliée utilise des images fausses (enfants aux mains coupées, par exemple) pour dire une réalité : le réel massacre de quelque 6500 civils belges et français. La différence est évidemment de taille. Cf. Laurence VAN YPERSELE (dir.), Questions d'histoire contemporaine : conflits, mémoires et identités, Paris, PUF (Quadrige), 2006, pp.169-189.

2 Cf. F. MORIMONT \& J.-L. VELLUT, op. cit., pp.155-165. 
fois des Blancs et des indigènes s'organisent le plus souvent autour des représentations de la mission civilisatrice coloniale : hiérarchisation des personnages, paternalisme, jeux de lumière désignant le bien, etc.

L'histoire de la Première Guerre mondiale aussi a été considérablement enrichie par l'analyse de la photographie. Il est vrai qu'il existe des centaines de milliers de clichés concernant cet événement hors norme et vécu comme tel par les contemporains. En effet, ces innombrables photographies disent à la fois l'horreur de la guerre et le patriotisme, la banalité de la vie au front et son infinie tristesse. Prenons l'exemple de la France : le service photographique de l'armée n'est créé qu'au printemps 1915. Autrement dit, avant cette date, les seules photos qui existent ont été prises par des amateurs. L'État, d'ailleurs, cherchera à les rassembler. Or, la création de ce service est motivée par une volonté de censure normale en temps de guerre, par un réel patriotisme, mais aussi par un souci de garder la mémoire de cet événement tel qu'il s'est passé. Pourtant, la censure sera loin d'être absolue : bien des soldats, malgré l'interdiction, montent au front munis d'un appareil photo. En outre, les journaux publient des clichés d'origines diverses : la monstration de la violence de guerre et de la mort au combat ira croissante à partir de 1916, ce qui correspond à un besoin de plus en plus pressant de « réaliser » la mort dans une société massivement en deuil ${ }^{1}$. Profitant de cette situation, un journal à sensation comme Le Miroir offre, en mars 1915, 30000 francs pour obtenir et éditer « la photo la plus saisissante » de la guerre ${ }^{2}$. Cela dit, les photos prises par les soldats sont assez différentes des autres ${ }^{3}$ : on y trouve peu de violence si ce n'est au travers des paysages en ruines et quasi pas de corps déchiquetés, mais bien de nombreux portraits de camarades au regard fatigué, des soldats lisant les lettres reçues de l'arrière (fig.7) ou s'adonnant à des activités ludiques. Ces photographes soldats qui vivaient constamment au milieu de la mort ont voulu photographier la vie. C'est le long temps de l'attente et l'invraisemblable banalité de la guerre qu'ils nous montrent. C'est l'ingéniosité des installations de fortune, les fêtes improvisées et la gueule

1 Cf. S. AUdOIN-ROUZEAU \& A. BECKER, 14-18, retrouver la guerre, Paris, Gallimard, 2000.

2 J. BEURIER, Images et violence 1914-1918. Quand Le Miroir racontait la Grande Guerre, Paris, Nouveau Monde, 2007. NB : 30000 Fr représente à l'époque 24 années de salaire d'un mineur!

3 Cf., par exemple, F. LACAILlE \& A. PetITEAU, Photographies de Poilus. Soldats photographes au cœur de la Grande Guerre, Paris, Somogy, 2004. 


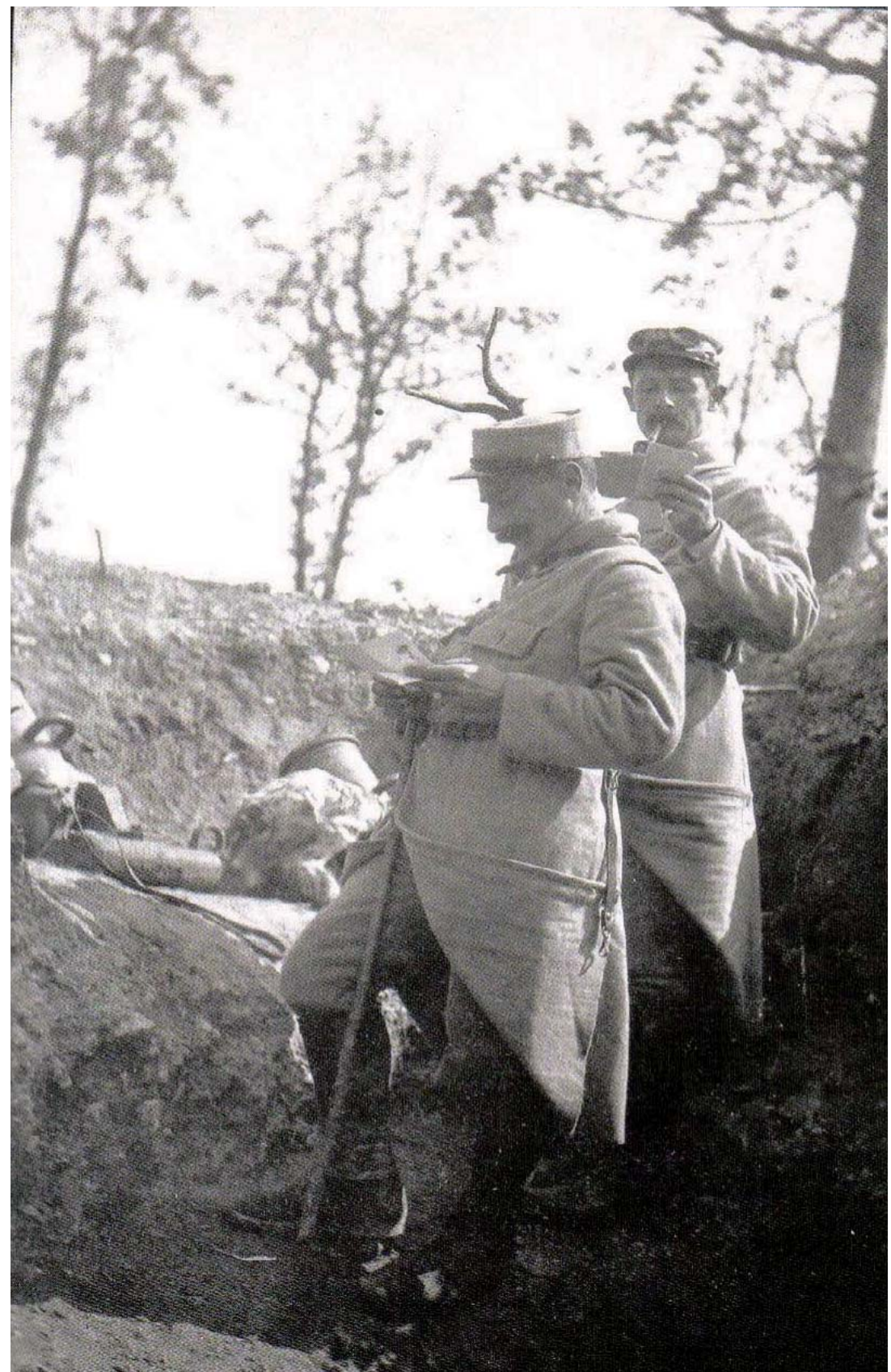

Fig.7. Photo prise par un soldat anonyme de camarades lisant leur courrier, Oise, 1915 (dans F. Lacaille \& A. Petiteau, Photographies de Poilus. Soldats photographes au coeur de la Grande Guerre, Paris, Somogy, 2004, p.75). 


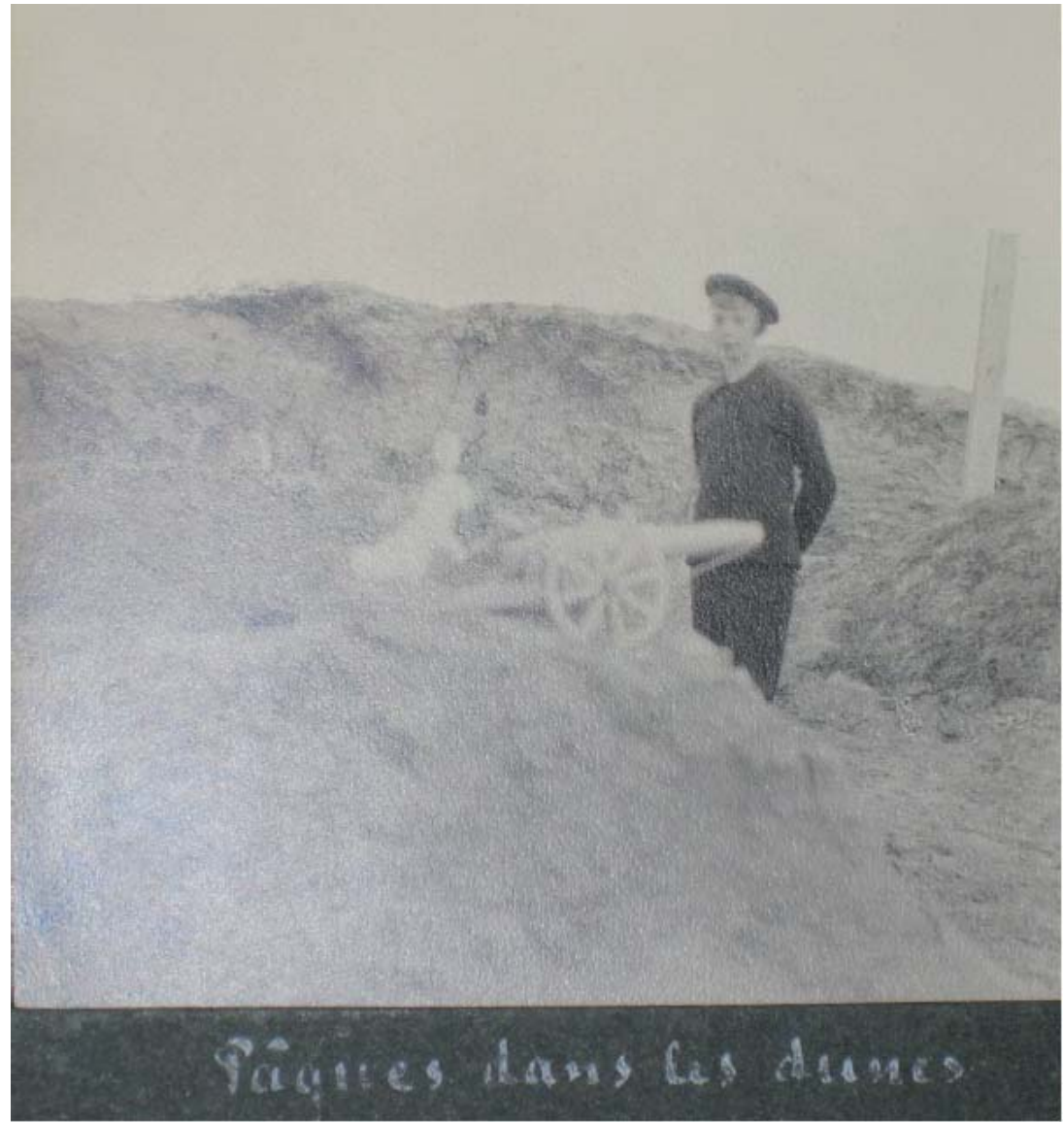

Fig.8. Photo prise par la reine Elisabeth, La Panne, Pâques 1915 (Bruxelles, Archives du Palais royal, Albums de la reine, 1914-1918, Vol.II, nº113, Pâques 1915).

des copains qu'ils fixent. C'est aussi les mille et une émotions humaines vécues durant cette guerre : tristesse, rire, fierté, inquiétude... Au fond, comme les soldats écrivains, ils ont tenté de dire l'indicible de cette Première Guerre mondiale qui oscille sans cesse entre horreur absolue et banalité quotidienne. La démarche de la reine Elisabeth, sur le front belge, est pratiquement la même : ses albums de guerre témoignent de la vie au front et non de la mort, de l'aide aux enfants et aux blessés, de la belle tenue des troupes que l'on passe en revue, des nouvelles armes et de la vie familiale à La Panne. Certaines photos montrent avec beaucoup de force l'irruption de la réalité de guerre dans la vie quotidienne, 
à moins que ce ne soit la persistance du quotidien dans la guerre. Ainsi, par exemple, la photographie du prince Léopold dans les dunes de La Panne avec, à l'avant-plan, un lapin de Pâques et un petit canon. Cette photo (fig.8) est prise en avril 1915, soit au moment même où le duc de Brabant s'engageait au $12^{\mathrm{e}}$ de Ligne comme simple soldat.

Bref, l'analyse des photographies, de leur thématique et de leur contenu, permet à l'historien d'approcher non seulement une certaine réalité du passé, mais surtout des imaginaires, des façons de voir et de vivre ce passé. Même les photos falsifiées ou les thématiques absentes en témoignent à leur façon. Au fond, pour peu qu'elle soit rigoureusement analysée et correctement recontextualisée tant en amont (contexte technique, mental, institutionnel) qu'en aval (question de la réception), la photographie est une trace particulièrement riche que l'historien ne peut ignorer. Par la diversité des supports, par l'abondance des réalisations, par la multiplicité de ses intentions, la photographie a littéralement envahi le monde contemporain. Sans compter que la puissance de la photographie a très largement façonné nos mémoires collectives comme nos imaginaires d'aujourd'hui. 
